\title{
Optimal Short-term Operation and Sizing of Pumped-Storage Power Plants in Systems with high Penetration of Wind Energy
}

\author{
Juan I. Pérez-Diaz, Alejandro Perea and José R. Wilhelmi, Member, IEEE
}

\begin{abstract}
In this paper the short-term optimal operation of an electric system comprising several thermal power plants and one pumped storage plant is studied in several scenarios of power demand and wind penetration in order to draw conclusions about the contribution of the pumped storage plant to system operation costs. A mixed integer linear programming model is used to obtain the optimal hourly thermal, hydro and pumping powers so that the production cost of the entire system is minimized. An aggregated piecewise linear hourly production cost curve is used to represent the thermal generation; the marginal production cost varying as a function of the power generated according to the slope of each piecewise linear segment. Main design parameters of the pumped storage plant are not considered fixed in the model but rather they are obtained in the solution with the purpose of drawing conclusions about the plant optimal sizing.
\end{abstract}

Index Terms-Pumped-storage power generation; Wind power generation; Power generation scheduling; Avoided costs; Mixed integer linear programming.

\section{NOMENCLATURE}

The notation used throughout the paper is stated as follows:

$A C^{\max }$ Maximum avoided cost (\%).

$c_{a}^{\min } \quad$ Aggregated thermal production cost corresponding to the minimum aggregated power $(\epsilon / \mathrm{h})$.

$c_{k}^{\min } \quad$ Production cost of thermal unit $k$ corresponding to its minimum power $(\epsilon / h)$.

$d_{h} \quad$ Binary variable used in the case that the the aggregated production cost curve is not strictly convex $\{0,1\}$.

$E_{B} \quad$ Pumping energy throughout the time horizon (GWh).

$E_{b}^{\max } \quad$ Maximum pumping energy among different wind speed scenarios (GWh).

$f$ Conversion factor $\left(0.0036 \mathrm{hm}^{3} / \mathrm{h} / \mathrm{m}^{3} / \mathrm{s}\right)$.

$I_{c} \quad$ Covariance index.

$K \quad$ Set of thermal generating units.

J. I. Pérez-Diaz and J. R. Wilhelmi are with the Department of Ilydraulic and Energy Engineering. Technical Univerșity of Madrid, c/ Profesor Aranguren $s / n, 28040$ Madrid (Spain) (e-mail: jiperez@caminos.upm.es; jrw(a)caminos.upm.es)

A. Perea is with the Department of Energy Management, Iberdrola Generación, c/ Tomás Redondo, l, 28033 Madrid (Spain) (e-mail: alejandro.perea@iberdrola.es).
$L^{a} \quad$ Set of linear segments of the aggregated piecewise linear thermal production cost curve.

$L^{h} \quad$ Set of linear segments of the flow-power curve of the pumped storage plant in generating mode.

$L_{k} \quad$ Set of linear segments of the production cost curve of thermal unit $k$.

$L^{p} \quad$ Set of linear segments of the flow-power curve of the pumped storage plant in pumping mode.

$p_{a}^{\min } \quad$ Minimum aggregated thermal power (MW).

$P_{D} \quad$ Power demand (MW).

$p d^{\text {max }}$ Maximum power demand (MW).

$p d^{\min } \quad$ Minimum power demand (MW).

$p d_{t} \quad$ Power demand at stage $t(\mathrm{MW})$.

$\overline{p h}$, Length of segment $l$ of the flow-power curve of the pumped storage plant in generating mode (MW).

$p h_{b} \quad$ Generating power at stage $t$ corresponding to segment $l$ of the flow-power curve of the pumped storage plant (MW).

$p h^{\text {min }}$ Minimum generating power of the pumped storage plant (MW).

$p h$ Generating power of pumped storage plant at stage $t$ (MW).

$p_{k} \quad$ Power output of thermal unit $k$ (MW).

$p_{k}^{\min } \quad$ Minimum power output of thermal unit $k$ (MW).

$\bar{p}_{i} \quad$ Length of segment $l$ of the aggregated piecewise linear thermal production cost curve (MW).

$p_{l k} \quad$ Power output of thermal unit $k$ corresponding to segment $l$ (MW).

$\bar{p}_{k} \quad$ Length of segment $l$ of the production cost curve of thermal unit $k(\mathrm{MW})$.

$p_{t}^{a} \quad$ Aggregated thermal power output corresponding to segment $l$ at stage $t(\mathrm{MW})$.

$\overline{p p}_{l} \quad$ Length of segment $l$ of the flow-power curve of the pumped storage plant in pumping mode (MW).

$p p_{h} \quad$ Pumping power at stage $t$ corresponding to segment $l$ of the flow-power curve of the pumped storage plant (MW).

$p p^{\text {min }}$ Minimum pumping power of the pumped storage plant (MW). 
$p p_{\ell} \quad$ Pumping power of pumped storage plant at stage $t$ (MW).

$p_{i}^{a} \quad$ Aggregated thermal power at stage $t(\mathrm{MW})$.

$p w_{t} \quad$ Wind power at stage $t(\mathrm{MW})$.

$\overline{p w} \quad$ Mean wind power (MW).

$q h^{\text {min }}$ Minimum generating water flow $\left(\mathrm{m}^{3} / \mathrm{s}\right)$.

$q h_{t} \quad$ Generating water flow at stage $t\left(\mathrm{~m}^{3} / \mathrm{s}\right)$.

$q p^{\text {min }}$ Minimum pumping water flow $\left(\mathrm{m}^{3} / \mathrm{s}\right)$.

$q p_{t} \quad$ Generating water flow at stage $t\left(\mathrm{~m}^{3} / \mathrm{s}\right)$.

$r_{l}^{a} \quad$ Slope of segment $l$ of the aggregated piecewise thermal production cost curve $(\epsilon / \mathrm{h} / \mathrm{MW})$.

$r_{l}^{h} \quad$ Slope of segment $l$ of the flow-power curve of the pumped storage plant in generating mode $\left(\mathrm{m}^{3} / \mathrm{s} / \mathrm{MW}\right)$.

$r_{i k} \quad$ Slope of segment $l$ of the production cost curve of thermal unit $k(\epsilon / \mathrm{h} / \mathrm{MW})$.

$r_{i}^{p} \quad$ Slope of segment $l$ of the flow-power curve of the pumped storage plant in pumping $\operatorname{mode}\left(\mathrm{m}^{3} / \mathrm{s} / \mathrm{MW}\right)$.

$T \quad$ Set of hourly stages of time horizon.

$u_{k} \quad$ Binary variable to decide whether or not to dispatch thermal unit $k\{0,1\}$.

$V^{i} \quad$ Initial volume in the upper reservoir $\left(\mathrm{hm}^{3}\right)$.

$V_{R} \quad$ Operational volume of the upper reservoir $\left(\mathrm{hm}^{3}\right)$.

$V_{R}^{\max } \quad$ Maximum operational volume of the upper reservoir $\left(\mathrm{hm}^{3}\right)$.

$v$ Volume in the upper reservoir at stage $t\left(\mathrm{hm}^{3}\right)$.

$x_{t} \quad$ Binary variable indicating whether or not the pumped storage is pumping at stage $t\{1,0\}$.

$y$ Binary variable indicating whether or not the pumped storage plant is generating at stage $t\{1,0\}$.

$\sigma \quad$ Standard deviation of the demand.

\section{INTRODUCTION}

$\mathrm{T}$ he intermittent nature of wind generation has become an issue of major concern in systems with high penetration of this renewable energy source. This subject reaches critical levels in isolated systems, as those existing in islands not interconnected to other islands or to the continent. Operational limits to the penetration of non-dispatchable energy sources make more difficult the substitution of fossil generation by renewable energies.

Adding pumped storage plants (PSP) to the system can compensate to some extent the time-shifting between wind generation and demand profiles, storing the wind energy that can not be absorbed by the system in low demand periods and making it available for peak periods. Additionally, it can introduce some degree of smoothing in the demand curve to be supplied by conventional power plants, shifting part of the higher cost generation in peak periods to lower cost valley periods.

In the last years these aspects have been studied by several researchers. In [1] an optimization approach is applied to the operation of a wind-hydro pumping storage power plant, considering the requirements of storage capacity. In [2] the same authors propose a similar model including the stochastic characteristics of the wind power. The market environment is considered in [3], where a methodology is presented to obtain the optimal bids for the day-ahead market. The uncertainties about prices and wind power are included, as well as the influence of the pumped storage size. Isolated systems have also been analyzed by several groups. Kaldellis et al. [4] present a parametric analysis of a combined wind-hydro plant in a medium size island of the Aegean Archipielago. In [5] a pumped hydro storage system is proposed to increase the penetration limits of wind power in Canary Island. Finally, in [6] the economic feasibility of a PSP is studied in an isolated system with high thermoelectric production and wind energy rejection.

This paper addresses the short-term (weekly) optimal operation of an electric system comprising several thermal power plants and one PSP with high levels of wind power penetration with the aim of drawing conclusions about the optimal sizing and influence of the PSP in the electric system operation costs. For that purpose, a mixed integer linear programming model is proposed. The paper is organized as follows: firstly, the proposed mixed integer linear programming model is described in section III; then, in section IV, the model is applied in an example system in different demand and wind power scenarios, the results being reported and discussed; and, finally, main conclusions of the study are presented in section $\mathrm{V}$.

\section{METHODOLOGY}

A mixed integer linear programming model is proposed in this paper to obtain the optimal hourly thermal, hydro and pumping powers so that the production cost of the entire system is minimized. An aggregated piecewise linear hourly production cost curve is used to represent the thermal generation; the marginal production cost varying as a function of the power generated according to the slope of each piecewise linear segment.

Even though this approach corresponds to a centralized system, alternatively, the marginal cost for each value of demand could represent the market prices in an ideal wholesale market [Ref]. It is worth to note that in the latter there would be some sensitivity of the market prices with respect to the thermal generation.

\section{A. Instantaneous load dispatch of thermal units}

In order to obtain the aggregated production cost curve of the thermal generation, it is necessary to know in advance the maximum and minimum powers and to piecewise linearize the production cost curve of each thermal generating unit. Then, an instantaneous load dispatch problem is solved by mixed integer programming to obtain the aggregated production cost of all thermal generating units of the system, the formulation of the problem being as follows:

$$
\min _{p_{l k}, u_{k}} \sum_{k \in K}\left(u_{k} c_{k}^{\min }+\sum_{l \in L_{k}} r_{l k} p_{l k}\right)
$$


subject to:

$$
\begin{gathered}
p_{k}=u_{k} p_{k}^{\min }+\sum_{l \in L_{K}} p_{l k} ; \forall k \in K \\
p_{l k} \leq u_{k} \bar{p}_{l k} ; \forall k \in K ; \forall l \in L_{k} \\
\sum_{k \in K} p_{k}=P_{D}
\end{gathered}
$$

where constraints (2) set the minimum power of each unit; constraints (3) set the maximum length of the segments of the linearized production cost curves; and constraint (4) forces the solution to supply the specified power demand level, $P_{D}$. The aggregated production cost curve of the thermal generation is obtained by solving the problem for each power demand level. Since this curve will be used within a weekly scheduling model, also based in mixed integer linear programming, it should be piecewise approximated by an appropriate number of linear segments.

\section{B. Optimal weekly scheduling model}

Once the aggregated production cost curve has been obtained, the optimal short-term operation schedule of the entire system (pumped storage and aggregated thermal plant) is obtained by solving a mixed integer linear programming problem, whose formulation is as follows:

$$
\min _{p_{t}^{a}, p h_{t}, p p_{t}} \sum_{t \in T}\left(c_{a}^{\min }+\sum_{i \in L^{a}} r_{l}^{a} p_{t h}^{a}\right)
$$

subject to:

$$
\begin{gathered}
p_{t}^{a}=p_{a}^{\min }+\sum_{t \in L^{a}} p_{t t}^{a} ; \forall t \in T \\
p_{l t}^{a} \leq \bar{p}_{l}^{a} ; \forall l \in L^{a} ; \forall t \in T \\
p_{t}^{a}+p h_{t}-p p_{t}=p d_{t}-p w_{t} ; \forall t \in T \\
v_{t}=v_{t-1}+\left(q p_{t}-q h_{t}\right) f ; \forall t \in T \\
q p_{t}=x_{t} q p^{\min }+\sum_{l \in L^{p}} r_{l}^{p} p p_{l t} ; \forall t \in T \\
p p_{t}=x_{t} p p^{\min }+\sum_{t \in L^{p}} p p_{l t} ; \forall t \in T \\
p p_{t} \leq x_{t} \overline{p p}_{l} ; \forall l \in L^{p} ; \forall t \in T \\
q h_{t}=y_{t} q h^{\min }+\sum_{i \in L^{h}} r_{l}^{h} p h_{l t} ; \forall t \in T \\
p h_{t}=y_{t} p h^{\min }+\sum_{t \in L^{h}} p h_{t t} ; \forall t \in T \\
p h_{t t} \leq y_{t} \overline{p h}_{l} ; \forall l \in L^{h} ; \forall t \in T \\
x_{t}+y_{t} \leq 1 ; \forall t \in T \\
v_{0}=V^{i} \\
v_{T} \geq v_{0}
\end{gathered}
$$

where constraints (6) set the minimum aggregated thermal power; constraints (7) set the maximum length of the segments of the linearized aggregated production cost curve; constraints (8) force the solution to supply the specified power demand level; constraints (9) express the water balance in the upper reservoir (an analogous set of constraints could be included so as to track the evolution of the lower reservoir, if necessary); constraints (10) to (12) express the relationship between the flow and power when the pumped-storage plant is pumping; constraints (13) to (15) express said relationship when the pumped-storage plant is generating; constraints (16) prevent the pumped-storage plant from simultaneously generating and pumping; and, finally, constraints (17) and (18) indicate that the initial volume in the upper reservoir is assumed to be known and that the final volume in the upper reservoir volume is forced to be greater or equal than the former, thus ensuring that only the water volume pumped from the lower reservoir will be used for energy generation.

It is worth to mention that, according to (5) and (6), the proposed formulation assumes that in no case there is enough wind power $\left(p w_{1}\right)$ to supply all the power demand by itself

It is important to note that, in the case that the piecewise linear approximation of the aggregated production cost curve is not strictly convex, constraints (7) should be replaced by constraints (19) to (21).

$$
\begin{gathered}
p_{l t} \leq d_{l t} \bar{p}_{l}^{a} ; \forall l \in L^{a} ; \forall t \in T \\
p_{l t} \geq d_{l+1, i} \bar{p}_{l}^{a} ; \forall l=1, \ldots, L^{a}-1 ; \forall t \in T \\
d_{l t} \geq d_{l+1, l} ; \forall l=1, \ldots, L^{a}-1 ; \forall t \in T
\end{gathered}
$$

In a similar way, if the relationship between flow and power in pumping or generating mode could lead in some cases to solutions where the use of water resulted artificially advantageous, then constraints (12) or (15) should be replaced by two/one set of constraints analogous to (19)-(21).

Finally, it is also important to point out that certain design parameters of the PSP, such as the maximum pumping and generating powers and the operational volume of the upper reservoir, are obtained as a solution of the model. For that purpose, the length of the last segment of the flow-power curve both in pumping and generating mode, is set great enough to prevent "saturation"; the needed operational volume in the upper reservoir is calculated as the difference between the maximum and minimum volumes obtained as the solution of the model.

\section{CASE STUDY}

In order to illustrate the applicability of the proposed model, it has been applied in an example system elaborated with realistic data taken from different sources. Thermal generation data, as well as the methodology used to obtain the production cost curve of each thermal generating unit, have been taken from $[7,8]$ and correspond to the system of Gran Canaria island. The system is composed of 4 gasoil, 5 diesel, 7 fuel oil and 1 combined cycle power plants, with capacities ranging from 12 to $210 \mathrm{MW}$ (all units except the combined cycle one have capacities lower than $80 \mathrm{MW}$ ). Fuel and gas prices have been taken from $[9,10]$. The aggregated production cost curve of the system has been piecewise approximated by eight linear segments. 
The proposed model has been applied in several demand and wind speed scenarios. Demand data have been taken from the web page of the Spanish Market Operator (OMEL) [11] and correspond to mainland electricity demand of the year 2008. Four different demand scenarios (Dscl-4) have been analyzed (see Fig. 1), each corresponding to a different season of the year 2008, and each properly scaled according to the generation capacity of the island. Wind speed data have been taken from HOMER software package, developed at the US National Renewable Energy Laboratory [12]. Four different wind speed scenarios (WSscA-D) have been analyzed for each above-mentioned demand scenario (see Fig. 2). In order to study different levels of wind power penetration, each wind speed scenario has been scaled to six different wind power levels (50-100-150-200-250-300-350 MW).

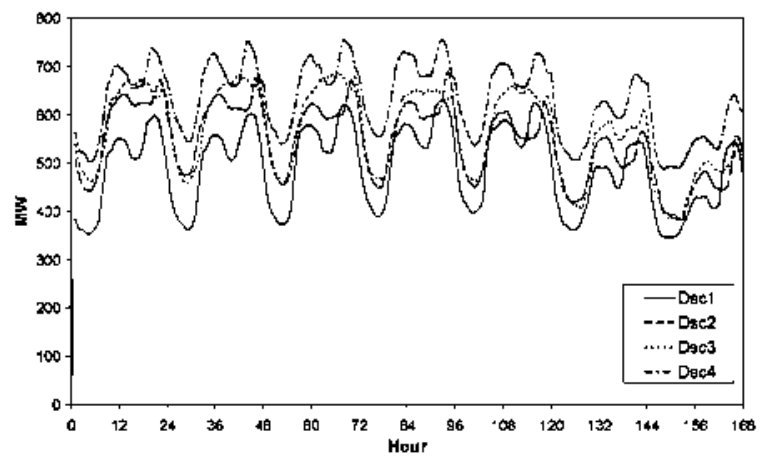

Fig. 1: Demand scenarios.

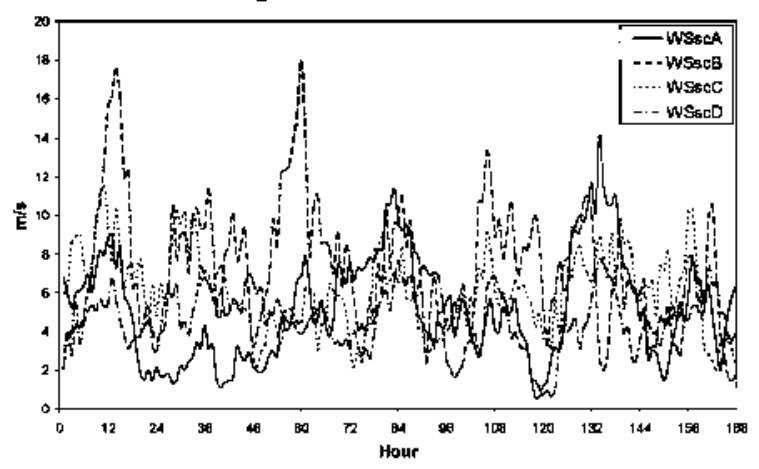

Fig. 2: Wind speed scenarios.

All cases have been solved using the CPLEX solver [13] under GAMS, in a Dell PWS 390 Intel dual-core $2.40-\mathrm{GHz}$ processor with $2.00 \mathrm{~GB}$ of RAM, with data and results import and export from and to MS Excel.

The system operation schedules obtained for the demand scenario Dsc4 and the wind speed scenario WSscA scaled to 150 and $300 \mathrm{MW}$ of wind power are shown in Figs. 3 and 4. In these figures it can be seen the peak showing effect of the PSP, especially in the second case, where the wind power reaches $300 \mathrm{MW}$ in several stages.

In all cases analyzed, the resulting operation costs have been compared with those of supplying the net demand only with the thermal units $(D C T)$ so as to calculate the reduction in production costs (avoided costs) caused by the PSP. In the cases shown in Figs. 3 and 4, the avoided costs are 0.17 and $0.44 \%$ (15,515 and $36,753 €)$, respectively. The avoided costs have been translated into annual recovered costs $(A R C)$ in all cases analyzed, assuming certain specific initial costs taken from [6], such as: $400,000 \mathrm{\epsilon} / \mathrm{MW}$ for pumping capacity; $500,000 \mathrm{\epsilon} / \mathrm{MW}$ for generating capacity; and $2 \mathrm{\epsilon} / \mathrm{m}^{3}$ for the upper reservoir size. In the cases shown in Figs. 3 and 4, the hydro producer would recover in a year the 0.65 and $1.06 \%$ of the initial investment cost, respectively

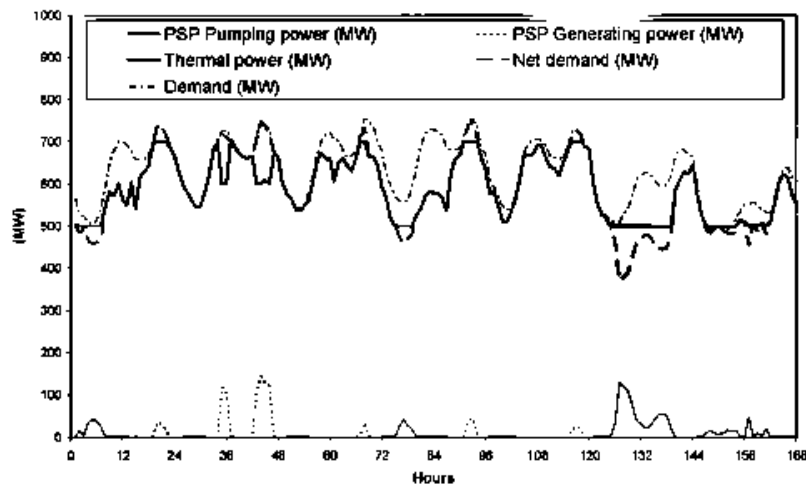

Fig. 3: System operation schedule for Dsc4 and WSscA (150 MW).

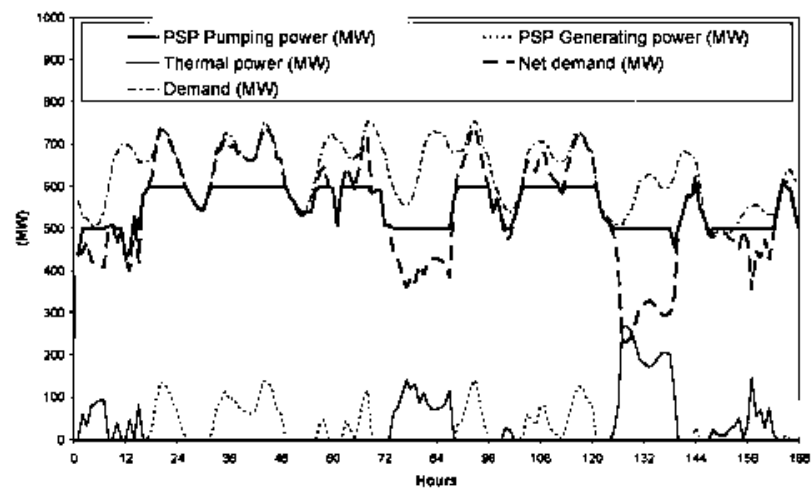

Fig. 4: System operation schedule for Dsc4 and WSscA (300 MW).

By comparing Figs. 3 and 4 one can observe that, as wind power increases, the contribution of the PSP to the system operation also increases. Nevertheless, this fact has not occurred in all cases analyzed, as it can be seen in the figures embedded in Table I, where the PSP pumping energy is plotted in solid line; the PSP generating energy is plotted in dashed line; the avoided costs are plotted in dotted line; and the upper reservoir volume is plotted in dash-dotted line, all against the wind power in the $x$-axis (the left $y$-axis is used for energy and the y-right axis is used for avoided costs and upper reservoir volume). Actually, in some cases, such as Dsc2WSscA or Dsc3-WSscB both the PSP pumping and generating energies monotonically decrease as wind power increases. By contrast, the avoided costs monotonically increase as wind power increases in all cases analyzed, with only very slight decreases $(<0.009 \%)$ in few cases between 50 and $100 \mathrm{MW}$ of wind capacity. As it can be seen in Table $I$, the maximum avoided costs range from 0.31 to $2.88 \%$ of $D C T$.

In order to identify relations between the results and the demand and wind speed scenarios, certain parameters have been included in Table $I$, such as the ratio peak/off-peak power demand $(P O P r)$, the per unit standard deviation $(s d)$ of the demand and an index, $I_{c s}$, indicating the covariance [14] between demand and wind power; the later being given by the 
TABLE I. Summary of results

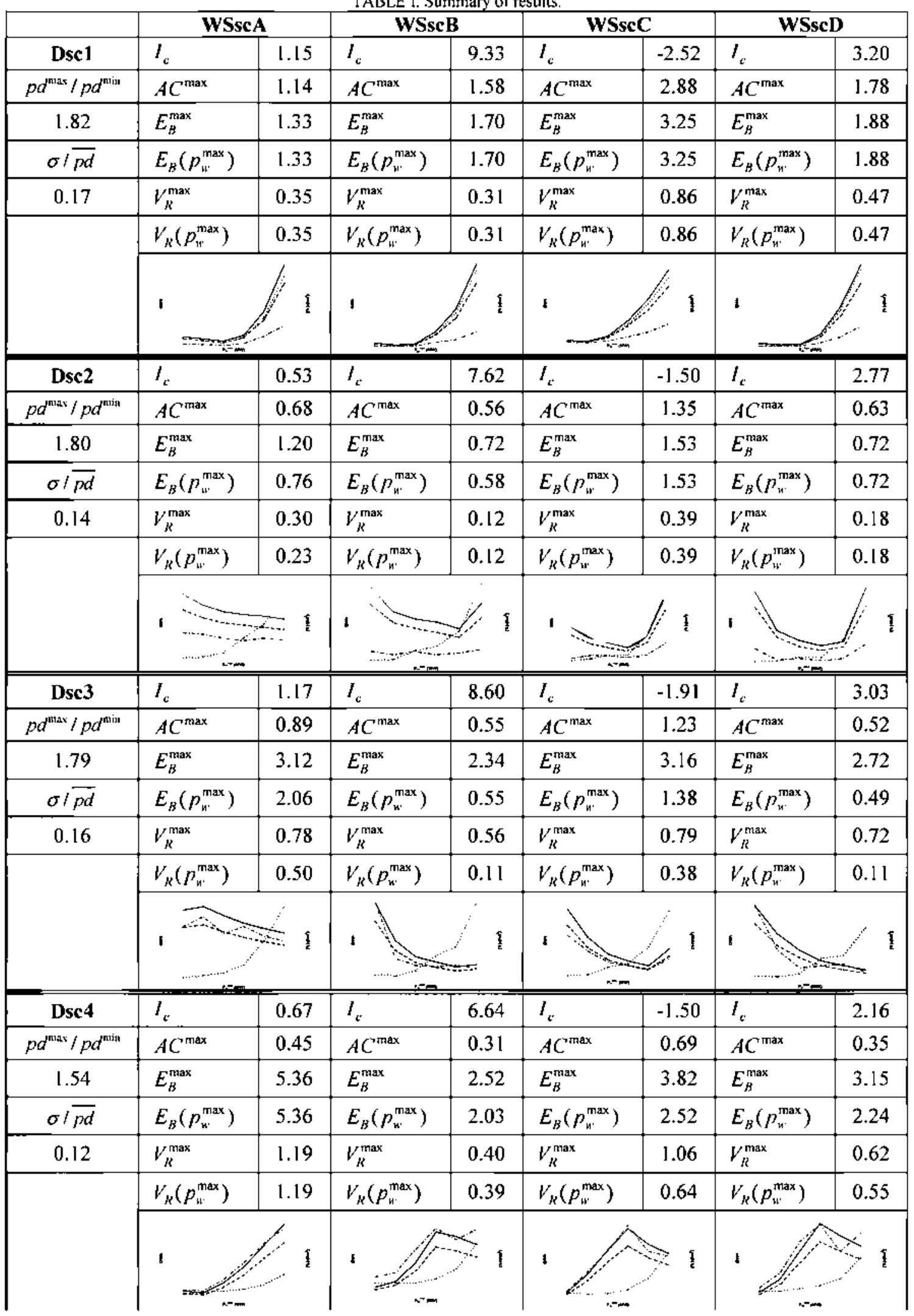

following formula:

$$
I_{c}=\sum_{i \in T}\left(\left(p w_{t}-\overline{p w}\right) / \overline{p w}\right)\left(\left(p d_{t}-\overline{p d}\right) / \overline{p d}\right)
$$

From the obtained results, it is possible to state that for a given demand, the maximum avoided costs are obtained in the wind power scenario with lower covariance. In this sense, it is important to emphasize the contribution of the wind power profile to the covariance index, as it can be seen by comparing the demand and wind speed profiles shown in Figs. 1 and 2. In 
an analogous way, it is possible to state that, in outline, for a given wind power scenario the avoided costs are higher in demand scenarios with higher $P O P r$ and $s d$ values (Dsc1-2-3).

Regarding the $A R C$, it follows in general a trend similar to that of the avoided costs, i.e. increasing as wind power increases. However, it is important to take into account that this parameter depends on the maximum pumping and generating powers, obtained as a solution of the model. This may cause $A R C$ to follow a different trend as for Dsc4, where: in WSscB-C-D, it decreases for wind powers lower than 150 or $200 \mathrm{MW}$ (depending on the case) and increases for higher wind powers; and in WSscA, it monotonically decreases as wind power increases. In a similar way, in Dsc1-WSscD and Dsc3-WSscD the avoided costs are respectively 1.78 and 0.52 $\%$; whereas the $A R C$ are, respectively, 2.38 and $3.19 \%$ of the investment costs. It is important to consider that, firstly, the $D C T$ are, respectively, 4.5 and $6.0 \mathrm{M} €$ and that, secondly, the maximum pumping powers are 229 and $72 \mathrm{MW}$. Therefore, in the PSP planning phase, it might be helpful to carry out a study similar to the one presented in this paper, trying to cover as many cases of wind power versus demand as possible or, at least, the most representative ones.

Finally, it is interesting to note that in all cases analyzed the PSP contributes to decrease and increase, respectively, the maximum and minimum powers supplied by the thermal units, the effect being more noticeable as the wind power increases (see Fig. 5). This fact could help, on one hand, to diminish, or avoid, wind power curtailments and, on the other hand, to reduce the greenhouse gases emissions of the system.

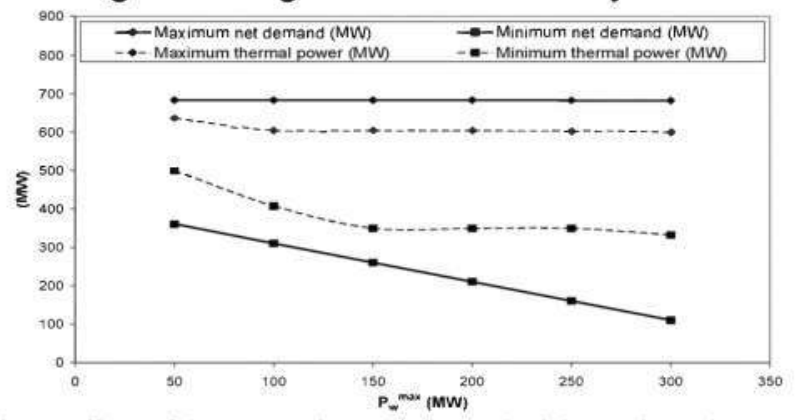

Fig. 5: Effects of the PSP on the maximum and minimum thermal powers.

\section{CONCLUSION}

In this paper, the contribution of a PSP to the operation of an electric system with high wind penetration has been studied with the help of a weekly optimal operation scheduling model based on mixed integer linear programming. An example system, elaborated from realistic data taken from different sources, has been used to test model. The system operation has been studied under different demand and wind speed scenarios, the later being scaled to different wind penetration levels. It has been shown that the PSP contributes in all cases analyzed to the reduction of the system operation costs, the magnitude of the reduction increasing as the level of wind power penetration increases. In addition, it has been shown that the PSP contributes as well to decrease and increase, respectively, the maximum and minimum powers supplied by the thermal units, what could help to avoid wind curtailments and to reduce the greenhouse gases emissions.
The methodology presented in this paper could serve as a basis for economic feasibility studies of pumped-storage facilities and even to help authorities to incentive the erection of such type of plants, establishing the adequate regulations and economical remunerations for the services rendered to the electric system.

\section{REFERENCES}

[1] E. D. Castronuovo and J. A. Peças Lopes, "Optimal operation and hydro storage sizing of a wind-hydro power plant", Electrical Power and Energy Systems, vol. 26, pp. 771-778, 2004.

[2] E. D. Castronuovo and J. A. Peças Lopes, "On the optimization of the daily operation of a wind-hydro power plant," IEEE Trans. Power Syst., vol. 19, pp. 1599-1606, 2004.

[3] J. Garcia-González, R. Moraga, L. Matres and A. Mateo, "Stochastic joint optimization of wind generation and pumped-storage units in an electricity market" IEEE Trans. Power Syst., vol. 23, pp. 460-467, 2008.

[4] J. K. Kaldellis, K. Kavadias and E. Christinakis, "Evaluation of the windhydro energy solution for remote Islands", Journal of Energy Conversion and Management, vol. 42(9), pp. 1105-1120, 2001

[5] C. Bueno, and J. A. Carta, "Wind powered pumped hydro storage systems, a means of increasing the penetration of renewable energy in the Canary Islands". Renewable and Sustainable Energy Review, vol. 10(4), pp. 312-340, 2006.

[6] D. A. Katsaprakakis, D. G. Christiakis, A, Zervos, D. Papantonis and S. Voutsinas, "Pumped storage systems introduction in isolated power production systems", Renewable Energy, vol. 33, pp. 467-490, 2008.

[7] Spanish Ministry of Industry, Tourism and Trade. Order ITC/913/2006, March $30^{\text {th }}$.

[8] Spanish Ministry of Industry, Tourism and Trade. Order ITC/914/2006, March $30^{\text {th }}$.

[9] Spanish Ministry of Industry, Tourism and Trade. Resolution November $26^{\text {th }}, 2007$

[10] Spanish Ministry of Industry, Tourism and Trade. Resolution September $4^{\text {th }}, 2008$.

[11] Operador del Mercado Ibérico de Energía - Polo Español, S.A. [Online]. www.omel.es

[12] U.S. Department of Energy. National Renewable Energy Laboratory, HOMER, Getting started guide, May 2003.

[13] CPLEX 10, in The Solver Manuals. Washington, D.C.: GAMS Development Corporation, 2006.

[14] M. Gopal, Modern control system theory, New Delhi: Wiley Eastern Limited, 1984

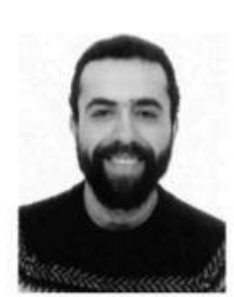

\section{BIOGRAPHIES}

Juan Ignacio Pérez-Díaz received the Ph.D. degree in engineering in 2008 from ETSICCP, Technical University of Madrid (UPM), where he is an assistant professor since 2009. His current research interests focus mainly on the operation and control of hydro power plants.

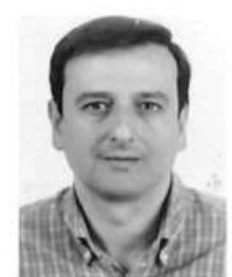

Alejandro Perea received the Engineering degree in 1981 from ETSICCP, Technical University of Madrid (UPM), Madrid, Spain. In 1982, he joined Instituto Tecnológico para Postgraduados, Madrid. In 1984, he joined Hidroeléctrica Española, that merged in 1990 to form Iberdrola, where he works until now. His current job and research interests are hydropower and energy systems management.

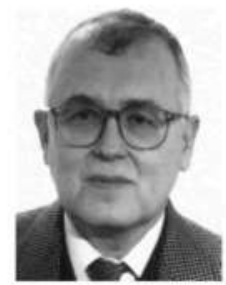

José Román Wilhelmi received the Ph.D. degree in engineering from ETSICCP, Technical University of Madrid (UPM), Madrid, Spain, in 1972. In 1974, he joined Empresarios Agrupados, where he worked in power plant engineering. In 1983, he became a Full Professor of electrical engineering at UPM. His present research interests are hydropower and other renewable energy sources, such as wind and marine currents or waves. 